\title{
INFLUENCE DE LA DÉSHYDRATATION A BASSE TEMPÉRATURE SUR LA VALEUR ALIMENTAIRE DES FOURRAGES
}

\author{
C. DEMARQUILLY \\ avec la collaboration de J, M. Boisseau et H. Bousquet \\ Station de Recherches sur l'Elevage des Ruminants, \\ Centre de Recherches de Clermont-Fervand, 63 -Saint-Genès-Champanelle \\ Institut national de la Recherche agronomique
}

\section{RÉSUMÉ}

I. A partir de 22 échantillons de fourrages verts (I 8 graminées et 4 trèfles violets) dont on mesurait la digestibilité et la quantité ingérée sur des lots de 6 moutons, nous avons préparé en 1967 et 1968, 22 fourrages déshydratés dans un séchoir Scolari dont la température était réglée entre 150 et $200^{\circ} \mathrm{C}$. Les 9 échantillons de 1967 ont été récoltés avec une machine à fléaux et les I 3 échantillons de 1968 fauchés à la barre de coupe ct ramassés aussitôt avec une ensileuse à pick-up et à couteaux. Les fourrages ont été pressés en ballots à la sortie de la déshydrateuse.

Nous avons en outre préparé 8 fourrages comprimés (Wafers) en agglomérant dans une presse à piston (Muller) $400 \mathrm{~kg}$ de 8 des 13 échantillons de 1968 . Cette opération a été effectuée après plusieurs mois cle conservation en ballots.

Durant l'hiver qui suivait leur récolte, nous avons mesuré la digestibilité et la quantité ingérée de ces fourrages déshydratés sur des lots de 6 moutons.

2. La déshydratation a provoqué une diminution faible et très peu variable cle la digestibilité de la matière organique des graminées; en moyenne - - I, I $\pm 0,9$ pour I7 échantillons. La digestibilité des matières azotées a en revanche été diminuée de 4,7 points soit 7,5 p. Ioo (tabl. I.) 60 p. I 00 de la diminution de la digestibilité de la matière organique est due à celle des matières azotées.

3. Par opposition aux graminées, la digestibilité cles 4 fourrages de trèfle violet a diminué beaucoup plus (tabl. I) : $\ldots 5,9 \pm 2,3$ points pour la matière organique et - I 6,4 points soit 25 p. Ioo pour les matières azotées. 55 p. Ioo de lat diminution de la digestibilité de la matière organique est due à celle des matières azotées.

4. Les 8 graminées déshydratées étudiées en 1967 ont été ingérées en quantités plus faibles (- I 5.4 p. roo) que les plantes sur pied correspondantes. Cette diminution semble résulter du fait que ces fourrages avaient été récoltés à l'ensileuse à fléaux car nous n'avons observé aucune diminution des quantités ingérées pour les graminées de 1968 récoltées avec une ensileuse à couteaux (tabl. I). Pour ces échantillons de I968, la quantité de matière organique digestible ingérée sous forme de produits déshydratés est la mème que celle ingérée avec les plantes sur pied correspondantes.

5. En revanche, pour les trèfles violets la quantité de matière sèche ingrérée à diminué de I 2 p. Ioo et la quantité de matière organique digestible ingérée do I9,o p. Ioo (tabl. I).

6. Le conditionnement des fourrages avec une presse à piston n'a pas provoqué de moditications significatives de la digestibilité et de la quantité ingérée (tabl. 2). En ce qui concerne la 
quantité ingérée, cela doit résulter du fait que les fourrages ont été comprimés longtemps après leur déshydratation alors qu'ils avaient repris de l'humidité. Leur passage dans la presse a donc provoqué un broyage beaucoup moins important que s'ils avaient été plus secs.

\section{INTRODUCTION}

La déshydratation des fourrages pour l'alimentation des ruminants connaît depuis ces trois dernières années un développement important en France.

Il existe encore très peu d'études sur les modifications de valeur alimentaire provoquées par la déshydratation; les seules études réalisées sont généralement anciennes et ont porté essentiellement sur les modifications de la digestibilité (Ėin et Helleday, I948; Watson, I95I ; Dijkstra, I957). Selon ces auteurs la déshydratation, quand elle a lieu avec des machines bien réglées, provoque une diminution négligeable (environ 2 points) de la digestibilité de la matière organique et une diminution plus importante (environ Io p. IOO) de la digestibilité des matières azotées.

Nous avons cherché à préciser ces pertes dans le cas de la déshydratation à basse température (entre I50 et $200{ }^{\circ} \mathrm{C}$ ) et avons étudié parallèlement l'influence que la (léshydratation en elle-même (c'est-à-dire sans le conditionnement du fourrage à la sortie du déshydrateur) pouvait avoir sur la quantité de fourrage ingérée par le mouton.

\section{MATÉRIEL E'T MÉTHODES}

A partir de 22 échantillons de fourrages verts $(5$ dactyles, 5 fétuques élevées, 5 ray-grass anglais, 3 ray-grass italicns ct + trèfles violets, en majorité du premier cycle de végétation) dont on mesurait la cligestibilité et la quantité ingéráe sur des moutons, nous avons fabriqué en 1967 et I 968,22 fourrages déshyclratés dans un séchoir Scolari dont la température était réglée entre I $50^{\circ}$ et $200{ }^{\circ} \mathrm{C}$. Les 9 échantillons de r 967 ont été récoltés avec une ensileuse à fléaux, et les I 3 échantillons de 1968 fauchés à la barre de coupe et aussitôt ramassés avec une ensileuse à pick-up et à couteaux (Scampolo). Ces fourrages ont été pressés en ballots dès leur sortie de la déshydrateuse.

Nous avons en outre fabriqué 8 fourrages comprimés en agglomérant dans une presse à piston (Muller) environ $400 \mathrm{~kg}$ de 8 des I 3 échantillons déshydratés en 1968. Cette opération a été effectuée en Novembre 1968, soit après plusieurs mois de conservation en ballots.

Durant l'hiver qui suivait leur récolte, chaque fourrage déshydraté a été distribué acl libitum (Io p. Ioo de refus) en 2 repas par jour à des lots de 6 moutons (4 en cage à métabolisme et 2 en stalles au sol) aussi semblables que possible à ceux qui avaient été utilisés pour les mesures sur les plantes sur pied. La méthode employée pour ces mesures a déjà été exposée dans des publications antérieures (Demarguilly et J Arikige, I964; Demarguilly, 1965).

\section{RÉSULTATS}

\section{Digestibilité}

A une exception près, la déshydratation a provoqué une diminution faible et très peu variable de la digestibilité de la matière organique des graminées ; en moyenne - I,I $\pm 0,9$ points pour I 7 échantillons. La digestibilité de la cellulose brute n'a pas été modifiée (augmentation non significative de $+0,5$ pcint) mais la digestibilité des matières azotées a été diminuée de $4,7 \pm 3,2$ points soit de 7,5 p. IoO (tabl. I). 


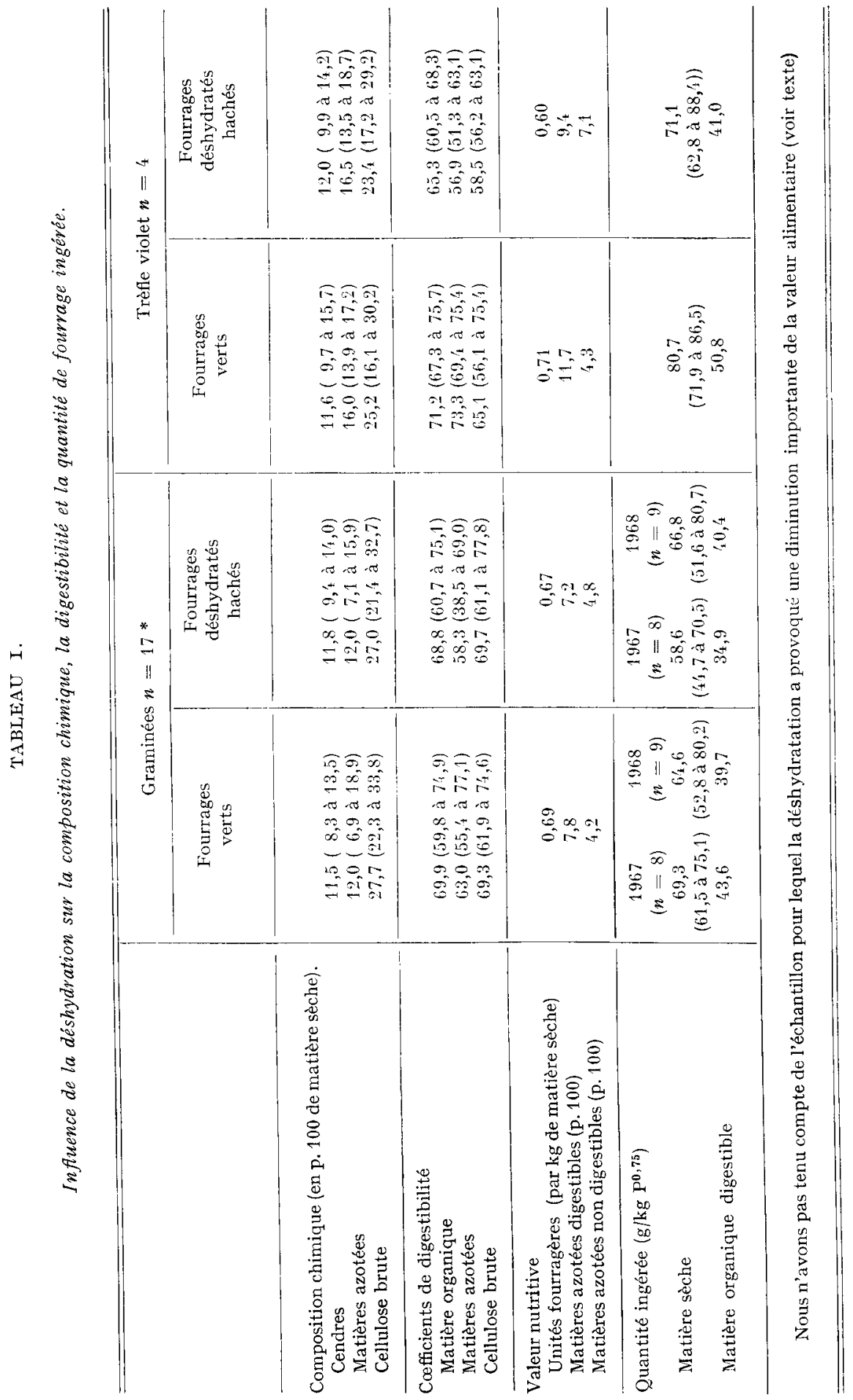


60 p. Ioo de la diminution de la digestibilité de la matière organique est due à celle des matières azotées. Pour un autre échantillon de graminées nous avons cepen dant observé une diminution importante de la digestibilité des différents constituants : respectivement - I 4,3-48,8 et - Io,5 points pour la matière organique, les matières azotées et la cellulose brute. Il s'agissait d'un échantillon de ray-grass italien Westerwold semé au printemps et exploité à un stade jeune (début de l'épiaison) et pauvre en matière sèche ( $44,0 \mathrm{p}$. IOO).

Par opposition aux graminées, la digestibilité des 4 fourrages de trèfle violet a diminué beaucoup plus (tab1. I) : $-\mathbf{5 , 9} \pm \mathbf{2 , 3}$ points pour la matière organique, $-6,6$ points pour la cellulose brute $-\mathrm{I} 4,6$ points (soit $22,4 \mathrm{p}$. Ioo) pour les matières azotées. La teneur en matières azotées non digestibles est passée de 4,3 p. Ioo pour les trèfles sur pied à $7, \mathrm{I} \mathrm{p}$. Ioo pour les trèfles déshydratés. Ces derniers étant légèrement plus riches en matières azotées que les échantillons sur pied correspondants, on peut estimer à $25 \mathrm{p}$. roo la diminution réelle de la digestibilité des matières azotées, puisqu'on sait que la digestibilité et la teneur en matières azotées sont étroitement liées et varient dans le même sens. 55 p. Ioo de la diminution de la digestibilité de la matière organique est due à celle des matières azotées.

La diminution de la digestibilité des différents constituants du trèfle violet, plus importante que celle enregistrée pour les graminées est due à une action directe de la déshydratation. Cela doit résulter du fait que le trètle violet, comme le ray-grass italien Westerwold, est une plante difficile à sécher, parce que riche en contenu cellulaire et en eau. La durée du séchage a donc été longue ce qui a dû entraîner un début de caramélisation.

\section{Quantités ingérées}

Les 8 graminées déshydratées étudiées en 1967 ont été ingérées en quantité plus faible que les plantes sur pied correspondantes : $-10,7 \mathrm{~g} / \mathrm{kg} \mathrm{P} \mathrm{P}^{0,75}$ soit $\mathrm{I} 5,4 \mathrm{p}$. Ioo. Cette diminution peut résulter soit d'un mauvais réglage de la déshydrateuse (mais qui n'a pas eu d'influence sur la digestibilité), soit plus vraisemblablement du fait que ces fourrages avaient été récoltés à l'ensileuse à fléaux. En effet en 1968 la récolte a été effectuée avec une récolteuse à couteaux et pour les $9 \mathrm{graminées} \mathrm{pour} \mathrm{lesquelles} \mathrm{la} \mathrm{déshy-}$ dratation n'a entraîné qu' une légère diminution de la digestibilité, nous n'avons observé aucune diminution de la quantité ingérée ; catte dernière a même été, mais de façon non significative, légèrement supérieure de $2,2 \pm 2,0 \mathrm{~g} / \mathrm{kg} \mathrm{P} \mathrm{P}^{\mathrm{j}, 75}$, soit $3,4 \mathrm{p}$. Ioo, ce qui est en accord avec les résultats obtenus sur vaches par VÉRITÉ (communication personnelle). Pour ces 9 échantillons, la quantité de matière organique digestible ingérée sous forme de produits déshydratés $\left(40,4 \mathrm{~g} / \mathrm{kg} \mathrm{P}^{0,75}\right)$ est pratiquement la même que celle ingérée avec les plantes sur pied correspondantes $(39,7 \mathrm{~g})$. Ein revanche, la quantité de matière sèche ingérée a été plus faible pour les trèfles déshydratés que pour les plantes sur pied correspondantes : - 9,6 $6 \pm \mathrm{I}_{4}, 8 \mathrm{~g} / \mathrm{kg} \mathrm{P}^{0,75}$ soit II,9 p. Ioo. Il en a été de même à fortiori de la quantité cle matière organique digestible ingérée : $-9,8 \mathrm{~g} / \mathrm{kg}$ $\mathrm{P}^{0,75}$ soit une diminution de I9,2 p. IOO (tabl. I).

\section{Influence du conditionnement}

Le conditionnement avec une presse à piston des fourrages déshydratés hachés n'a provoqué que des modifications très faibles et non significatives de la digestibilité 


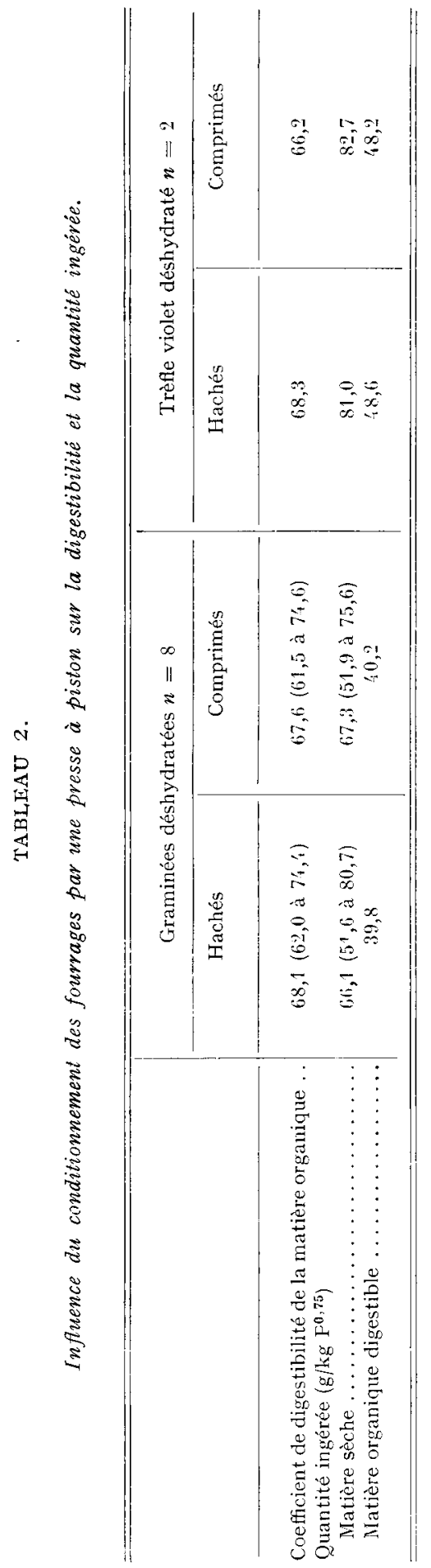


et de la quantité ingérée ; respectivement - o,5 $\pm \mathrm{I}, 0$ point et $+\mathrm{I}, 2 \pm 4,2 \mathrm{~g} / \mathrm{kg}$ $\mathrm{P}^{0,75}$ pour les 8 graminées (tabl. 2 ).

Il peut paraître étonnant que le conditionnement n'ait pas augmentéles quantités ingérées. Cela résulte essentiellement du fait que les fourrages ont été comprimés assez longtemps après leur déshydratation et qu'ils avaient repris de l'humidité. Leur passage dans la presse s'est donc accompagné d'un broyage beaucoup moins important que s'ils avaient été plus secs; ils contenaient en moyenne 55 p. Ioo de particules de taille supérieure à $1,25 \mathrm{~mm}$. Ils auraient, vraisemblablement, été un peu plus broyés et, par là, ingérés en plus grande quantité, s'ils avaient été pressés dès la sortie de la déshydrateuse.

Ces résultats montrent que la déshydratation à basse température effectuée dans de bonnes conđitions (séchoir bien réglé, fourrages récoltés avec une machine à coupe franche et se séchant facilement, ) diminue très peu la digestibilité de la matière organique des graminées et ne modifie pas la quantité de matière sèche ingérée et la quantité de matière organique digestible ingérée. La digestibilité, la quantité de matière sèche ingérée et la quantité de matière organique digestible ingérée (critère de la valeur alimentaire) des graminées déshydratées dépendront essentiellement de calles des plantes sur pied au moment de la fauche ( $\mathrm{r}$ est égal respectivement à 0,914 ; $0,95^{8}$ et 0,960 ) c'est-à-dire de l'espèce végétale et du stade de récolte. En revanchs, la déshydratation diminue de 7,5 p. Ioo la digestibilité des matières azotées. La terenr en matière azotées digestibles des graminées déshydratées à basse température pourra être obtenue en soustrayant 4,8 de la teneur en matières azotées. Cette valeur es 11 peu plus faible que celle que nous avons enregistrée avec la déshydratation à haute température; en moyenne 4,9 dans les meilleures conditions, 5,8 dans les conditions de la pratique.

En revanche, la déshydratation à basse température provoque des diminutions importantes de la digestibilité des différents constituants, de la quantité ingérée et de la valeur alimentaire des fourrages difficiles à sécher (trèfle violet, graminées exploitées l'année du semis).

Reçu pour publication en janvier 1970.

\section{SUMMARY}

EFFECT OF LOW TEMPERATURE-DEHYDRATION ON FORAGE FEED VALUE

I. Digestibility and fect intake were measured on lots of 6 shcep using 22 green forage samples (I8 grasses, 4 red clover) in 1967 and 1968 . We prepared 22 dehydrated forages in a Scolari drier, with the temperature fixed between 150 and $200^{\circ} \mathrm{C}$. The nine 1967 samples were harvested with a flail-type forage harvester and the thirteen I968 samples were eut with a reciprocating knife bar mower and gathered up immediately with a blade-type forage harvester. The forages were pressed into bales on leaving the drier.

We also prepared 8 wafers, agglomerating in a piston press (Muller) $400 \mathrm{~kg}$ of 8 of the thirteen I 968 samples. This was done after conservation in bale for several months.

We measured the digestibility and intake of these dehydrated forages on lots of 6 sheep in the winter following their harvest.

2. Dehydration caused a low, consistent decrease in the organic matter digestibility of the grasses : average of - I.I \pm 0.9 for I 7 samples. On the other hand, digestibility of crude protein was decreased by 4.7 points or 7.5 p. roo (table I). 60 p. roo of the digestibility decrease of organic matter was due to that of crude protein. 
3. As opposed to grasses, digestibility of the 4 red clover forages decreased much more (table I) : $-5.9 \pm 2.3$ points for organic matter and -16.4 points or 25 p. Ioo for crude potein. $55 \mathrm{p}$. IOo of the digestibility decrease of organic matter was due to that of crude potein.

4. The 8 dehydrated grasses studied in 1967 were ingested in much less quantity (- I 5.4 p. roo) than the corresponding standing plants in 1968 . This decrease may be because we harvested these forages with a flail-type harvester. No decrease in intake was noted for the 1968 grasses harvested with a blade-type harvester (table I). The amount of digestible organic matter ingested in a dehydrated form was the same as that ingested with the corresponding standing plants.

5. The dry matter intake for red clover decreased by 12 p. Ioo and the amount of digestible organic matter ingested by 19.0 p. Ioo (table I).

6. Conditioning the forages with a piston press did not cause significant modification in digestibility or intake (table 2). As far as the amount ingested is concerned, the forages must have been compressed a long time after dehydration, when they were damp. When pressed, they were not ground as much as if they had been dry.

\section{RÉFÉRENCES BIBLIOGRAPHIQUES}

Demarouilly C., I965. Factors affecting the voluntary intake of green forage by sheep. Proc. 9 th International Grassland Congress.

Demarquilly C., Jarkige R., ig64. Valeur alimentaire de l'herbe des prairies temporaires aux stades d'exploitation pour le pâturage. r. Composition chimique et digestibilité. Ann. Zootech., 13, 30 I-339.

Dijustra N. D., 1957. The conservation of grass for feeding purposes in Agriculture. Netherl. $J$. A gric. Sci., 5, 5, $27 \mathrm{I}-283$.

Edin H., Helleday T., I942. Undersökningar rörande näringsutbytet vid konsttorkning av vallväxter. Husd jursföröksanstaltens medd., 11, $38 \mathrm{pp}$.

Watson S. J., I95I. Grassland and grassland products. (London, I95 I). 